\title{
Application of Antifreeze Proteins for Sperm Cryopreservation
}

\author{
Saeed Zandiyeh ${ }^{1}$, Bita Ebrahimi ${ }^{2}$ and Marjan Sabbaghian ${ }^{1 *}$ \\ ${ }^{1}$ Department of Andrology, Reproductive Biomedicine Research Center, Royan Institute for Reproductive Biomedicine, Iran \\ ${ }^{2}$ Department of Embryology, Reproductive Biomedicine Research Center, Royan Institute for Reproductive Biomedicine, Iran
}

*Corresponding author: Marjan Sabbaghian, Reproductive Biomedicine Research Center, Royan Institute for Reproductive Biomedicine, No 12, Hafez St. Banihashem St, Resalat St, PO Box 19395-4644, ACECR, Tehran, Iran.

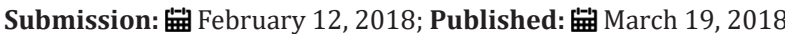

\section{Introduction}

Cryopreservation is an important technique used to store various types of cells, tissues, and organs at very low temperature, usually in liquid nitrogen $\left(-196{ }^{\circ} \mathrm{C}\right)$ [1]. However, freezing and thawing process causes cryo-injuries to cells. During cryopreservation creation of extracellular ice may cause rupture of the cell membrane and recrystallization of intracellular and extracellular ice may further damage the cells during the freezing and thawing process, respectively.

Many compounds act as cryoprotectants and are used for protection of cells against freezing. There are two types of cryoprotectants: (a) membrane permeating which can freely diffuse the membrane such as glycerol (G), ethylene glycol (EG) and dimethyl sulfoxide (DMSO); (b) non permeating membrane which cannot permeate the cell membrane such as sugars and antifreeze proteins [2]. Antifreeze proteins (AFPs) and Antifreeze glycoproteins (AFGPs) are produced by certain fish, insects, plants and bacteria that protect them in extremely cold climates from deep freezing temperatures [1]. AFPs act by decreasing the freezing point, modifying the ice-crystal formation process, preventing recrystallization and interacting with plasma membrane at low temperatures [3], thus allowing these species to survive in waters colder than the equilibrium freezing point of their internal fluids [4]. Antifreeze glycoproteins (AFGPs) are categorized into 8 classes of descending size from AFGP1 with Mw $=33.7 \mathrm{kDa}$ to AFGP8 with $\mathrm{Mw}=2.6 \mathrm{kDa}$ [5]. In their order of discovery, AFPs are sequentially numbered type I, II, III, and IV [6]. Early studies focused on AF(G) Ps isolated from natural sources mostly fishes. Nowadays most laboratories produce proteins and peptides either by recombinant expression in a suitable host like E. coli or solid-phase peptide synthesis [7,8]. The first application of marine AFPs to the protection of membranes at hypothermic temperatures was made in 1990 using AFGP from Antarctic and Arctic fishes [9]. Although most cryopreservation trials using AFPs have demonstrated that the addition of AFPs could improve post-thaw sperm viability, regardless of the freezing method, storage temperature, and biological sample, several reports showed no beneficial effect [10].
In the cryopreservation of cell lines, AFPs have been used as additives to conventional freezing medium to reduce the high amount of cytotoxic cryoprotectants (CPAs) and reduce freezing damage. One of the cell types that AFPs were tested for its cryopreservation was sperm cells [11]. Freezing-thawing process during cryopreservation is known to be detrimental to sperm function and ultimately to fertility. Cryopreservation promotes processes of capacitation, leakage of acrosomal content and induction of acrosomal reaction thus reducing sperm fertilization capacity in the reproductive tract. Previously, fish AFPs were studied for their efficiency when supplemented in sperm preservation media for different species at freezing and chilling stages [1].

In ram, chimpanzee, bovine, buffalo and fish, sperm quality was improved after cryopreservation and chilled storage by usage of AFPs [11]. Younis et al. [12] observed increased motility of chimpanzee sperm after freezing when AFPIII was used. Nevertheless, other studies reported better results with AFPI, such as Prathalingam et al. [13] AFPIII seems to have prevented the increase in the saturated fatty acid proportion during cryopreservation and hence would have interacted preferentially with unsaturated fatty acids. The membrane lipid composition dictates the protection conferred by the AFPs during chilling [14]. Nevertheless, as explained by Inglis et al. [15] AFPIII, in the cryopreservation solution decreases the loss of sperm quality and helps maintain the lipid composition of the plasma membrane according to the composition of the analyzed domains (head and flagella) during the freezing/ thawing process. Noteworthy, we used Antifreeze protein III in the human sperm freezing medium, and its results on sperm parameters are desirable, which will soon be published.

Finally, it is encouraging that many research groups studying AFP worldwide have started expanding their research into cryopreservation by using AFPs. We hope these concerted efforts will accelerate the development of biomedical application of AFPs.

\section{References}

1. Bang JK, Lee JH, Murugan RN, Lee SG, Do H, et al. (2013) Antifreeze peptides and glycopeptides, and their derivatives: potential uses in 
biotechnology. Mar Drugs 1(6): 2013-2041.

2. Pegg DE (2007) Principles of cryopreservation. Methods Mol Biol 368 39-57.

3. Fuller BJ (2004) Cryoprotectants: the essential antifreezes to protect life in the frozen state. Cryo Letters 25(6): 375-388.

4. Can 0, Holland NB (2013) Utilizing avidity to improve antifreeze protein activity: a type III antifreeze protein trimer exhibits increased thermal hysteresis activity. Biochemistry 52(48): 8745-8752.

5. Voets IK (2017) From ice-binding proteins to bio-inspired antifreeze materials. Soft Matter 13(28): 4808-4823.

6. Agarwal A, Mulgund A, Hamada A, Chyatte MR (2015) A unique view on male infertility around the globe. Reproductive Biology and Endocrinology 13: 37.

7. Lotze S, Versluis J, Olijve LL, van Schijndel L, Milroy LG, et al. (2015) Communication: Probing the absolute configuration of chiral molecules at aqueous interfaces. J Chem Phys 143(20): 201101.

8. Haymet A, Ward LG, Harding MM (1999) Winter flounder "antifreeze" proteins: synthesis and ice growth inhibition of analogues that probe the relative importance of hydrophobic and hydrogen-bonding interactions. Journal of the American Chemical Society 121(5): 941-948.

9. Rubinsky B, Arav A, Mattioli M, Devries AL (1990) The effect of antifreeze glycopeptides on membrane potential changes at hypothermic temperatures. Biochemical and biophysical research communications 173(3): 1369-1374.

10.Zilli, L, Beirão J, Schiavone R, Herraez MP, Gnoni A, et al. (2014) Comparative proteome analysis of cryopreserved flagella and head plasma membrane proteins from sea bream spermatozoa: effect of antifreeze proteins. PLoS One 9(6): e99992.

11. Qadeer S, Khan MA, Shahzad Q, Azam A, Ansari MS, et al. (2016) Efficiency of beetle (Dendroides canadensis) recombinant antifreeze protein for buffalo semen freezability and fertility. Theriogenology 86(7): 1662-1669.

12. Younis AI, Rooks B, Khan S, Gould KG (1998) The effects of antifreeze peptide III (AFP) and insulin transferrin selenium (ITS) on cryopreservation of chimpanzee (Pan troglodytes) spermatozoa. J Androl 19(2): 207-214.

13. Prathalingam N, Holt WV, Revell SG, Mirczuk S, Fleck RA, et al. (2006) Impact of antifreeze proteins and antifreeze glycoproteins on bovine sperm during freeze-thaw. Theriogenology 66(8): 1894-1900.

14. Tomczak MM, Vígh L, Meyer JD, Manning MC, Hincha DK, et al. (2002) Lipid unsaturation determines the interaction of AFP type I with model membranes during thermotropic phase transitions. Cryobiology 45(2): $135-142$.

15. Inglis SR, Turner JJ, Harding MM (2006) Applications of type I antifreeze proteins: studies with model membranes \& cryoprotectant properties. Current Protein and Peptide Science 7(6): 509-522.

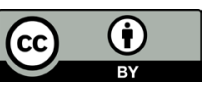

Creative Commons Attribution 4.0

International License

For possible submissions Click Here

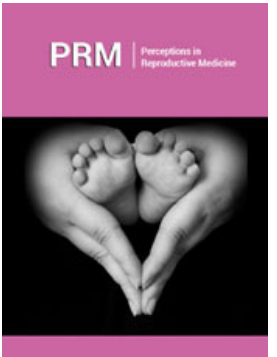

\section{Perceptions in Reproductive Medicine}

\section{Benefits of Publishing with us}

- High-level peer review and editorial services

- Freely accessible online immediately upon publication

- Authors retain the copyright to their work

- Licensing it under a Creative Commons license

- Visibility through different online platforms 